\title{
Estimación del crecimiento del jurel Trachurus murphyi capturado en el Perú, a partir del análisis de las frecuencias por tallas
}

\author{
Estimation of growth parameters of Jack mackerel Trachurus murphyi caught in Peru, from \\ length frequency analysis
}

\author{
Erich Díaz
}

Instituto del Mar del Perú, esquina Gamarra y General Valle S/N Chucuito, Callao, Perú.

Email Erich Díaz: ediaz@imarpe.gob.pe

Citación:

Díaz E. 2013. Estimación del crecimiento del jurel Trachurus murphyi capturado en el Perú, a partir del análisis de las frecuencias por tallas. En: Csirke J., R. Guevara-Carrasco \& M. Espino (Eds.). Ecología, pesquería y conservación del jurel (Trachurus murphyi) en el Perú. Rev. peru. biol. número especial 20(1): 061- 066 (Septiembre 2013)

\section{Resumen}

Se presentan las estimaciones de los parámetros de crecimiento del modelo de von Bertalanffy para el jurel Trachurus murphyi Nichols 1920 capturado en el mar peruano entre 1972 y 2012, a partir de análisis de frecuencias por tallas. La $L_{\infty}$ fue estimada a partir de la máxima talla observada a lo largo de la historia de su pesquería y se le mantuvo como parámetro fijo en la estimación del parámetro de crecimiento $k$ a partir del ajuste directo de una curva de crecimiento sobre la progresión mensual de modas. Los valores estimados fueron: $L_{\infty}=81.6$ $\mathrm{cm}$ y k $=0.167 \mathrm{año}^{-1}$. Se destaca que este valor de $\mathrm{k}$ es similar al estimado por otros autores y con otros métodos para el Perú, y es a su vez más alto que el estimado para el T. murphyi capturado frente a las costas de Chile. Estos resultados sugieren que el T. murphyi en el Perú tiene una velocidad de crecimiento más alta que el T. murphyi de Chile.

Palabras clave: Jurel, Trachurus murphyi, crecimiento, frecuencias por tallas, parámetros de crecimiento.

\section{Abstract}

This paper presents estimates of the von Bertalanffy growth parameters for Jack mackerel $T$. murphyi caught in Peruvian waters from 1972 to 2012, based on length frequency analysis. $L_{\infty}$ was estimated from the maximum length observed throughout the history of the fishery, and was kept as a fixed parameter in the estimation of $k$ obtained by fitting a growth curve to the monthly progression of modes. The estimated values were: $\mathrm{L}_{\infty}=81.6 \mathrm{~cm}$ and $\mathrm{k}=0.167 \mathrm{y}^{-1}$. It is highlighted that this $\mathrm{k}$ values is similar to those estimated by other authors for Peru, and is higher than those estimated for the T. murphyi caught off in Chile. These results suggest that T. murphyi caught in Peru has a faster growth rate than the T. murphyi from Chile.

Keywords: Jack mackerel, Trachurus murphyi, growth, length frequencies, growth parameters.

\section{Introducción}

Históricamente, la estimación de los parámetros de crecimiento del T. murphyi capturado en aguas peruanas se ha basado en la lectura de estructuras duras como los otolitos, y las lecturas y estimados han sido muy similares y las pequeñas diferencias pueden ser atribuidas principalmente a los periodo de estudio y las características de las muestras (Abramov \& Kotlyar 1980, Dioses 1995, Goicochea et al. 2013). Sin embargo, estas estimaciones para el jurel de aguas peruanas muestran marcadas diferencias con las hechas para el T. murphyi capturado frente a Chile (Kochkin 1994, Cubillos \& Arancibia 1995, Gili et al. 1995 en Serra \& Canales, 2009). La mayoría de estas diferencias son atribuibles a las diferentes interpretaciones que se da a los anillos de crecimiento de los otolitos (Dioses 2013a).

Los métodos basados en el Análisis de las Frecuencias por Tallas (AFT) se presentan como una alternativa metodológica aceptable para comparar y validar los parámetros estimados por la lectura de otolitos. Además de ofrecer una fuente de análisis independiente, el empleo del AFT permite el uso de información abundante y fácil de obtener, como son las frecuencias por tallas, y el menor tiempo requerido para las estimaciones ahora que se cuenta con mejores facilidades computacionales. Una desventaja de los métodos basados en el AFT son su alta sensibilidad a la baja representatividad de las
Publicado online: $\quad 11 / 10 / 2013$ Publicado impreso: $15 / 10 / 2013$ 


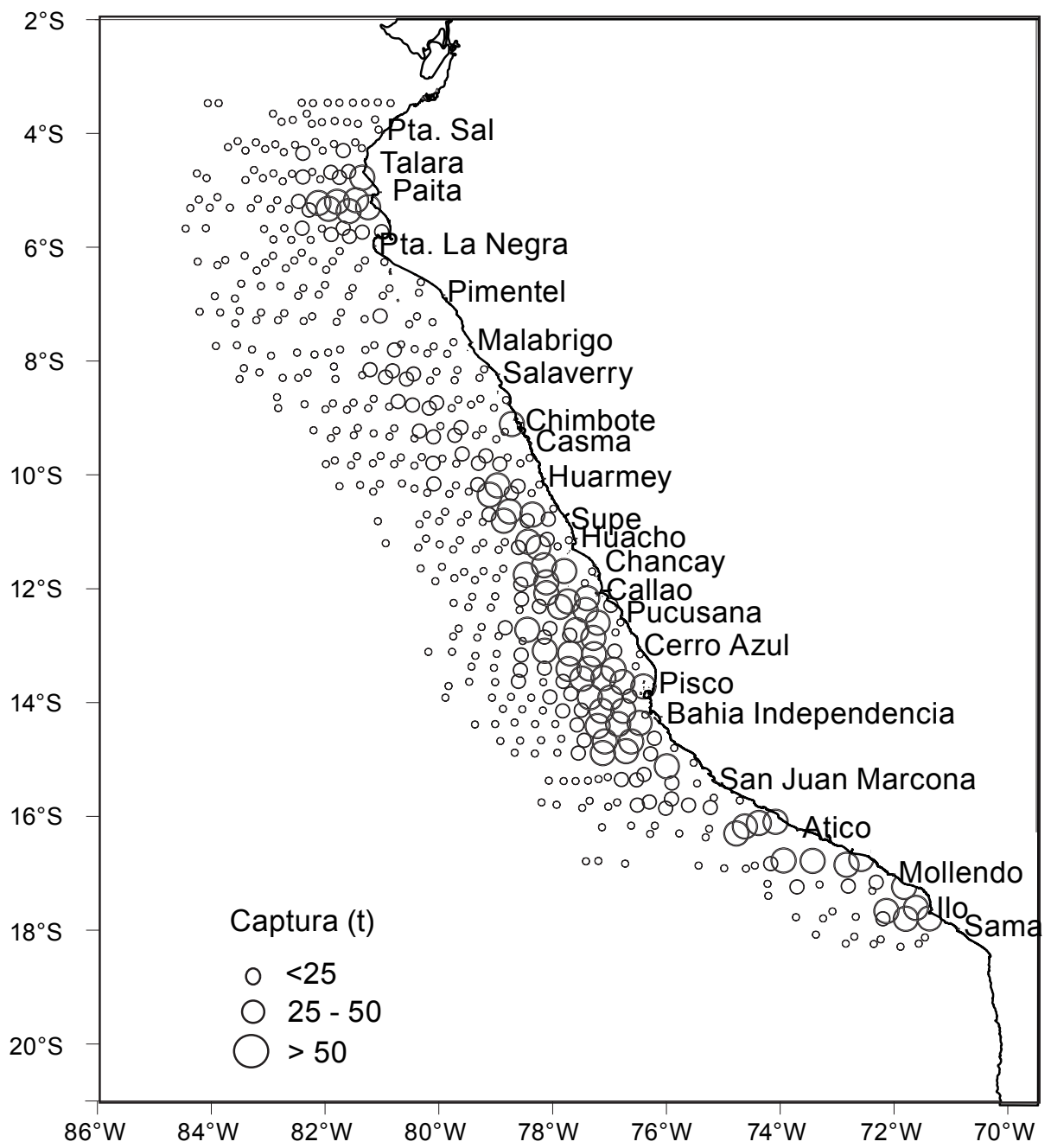

Figura 1. Distribución de las capturas a partir de las cuales se obtuvieron los muestreos biométricos, entre enero de 1972 y abril del 2012. Figure 1. Catch distribution from which biometric samplings were obtained between January 1972 and April 2012.

tallas extremas. Por lo general, las tallas próximas o debajo de la talla de reclutamiento suelen estar sub-representadas en los muestreos, mientras que las tallas más grandes también pueden estar poco representadas por problemas de disponibilidad o por ser escasas por efecto la mortalidad total acumulada. Otro aspecto que dificulta la aplicación de estos métodos es la ausencia de progresión modal en las frecuencias de tallas, como podría ocurrir por efecto de la selectividad en forma de domo o campana (Gulland 1987).

Se han propuesto varios métodos paramétricos y no paramétricos para estimar el crecimiento a partir del AFT (Pauly \& Morgan 1987). Para un grupo de ellos el razonamiento consiste en: 1) identificar un grupo modal objetivo en una frecuencia de tallas al inicio de la observación; 2) realizar un seguimiento temporal de la progresión en talla de dicho grupo; y 3) ajustar una curva de crecimiento que pase por la mayor cantidad de modas observadas. Para otro grupo de métodos el razonamiento consiste en: 1) identificar y extraer los grupos de edad contenidos en las frecuencias de tallas (i. e. pseudocohortes); 2) estimar las tallas medias de cada grupo extraído; y 3) ajustar un modelo de crecimiento a todos los pares de datos edad-talla disponibles. En términos generales, se ha observado que con un muestreo representativo de las tallas estos métodos producen buenos estimados de crecimiento (Gulland 1987).
En este contexto, el objetivo principal del presente trabajo es estimar los parámetros de crecimiento del T. murphyi capturado en aguas peruanas utilizando el AFT, considerando que contribuirá a mejorar el conocimiento sobre el crecimiento del T. murphyi en el Pacífico Sur. Para ello, se utilizó en esta oportunidad el primer enfoque descrito líneas arriba, como es el ajuste de curvas de crecimiento a progresiones modales. Se discuten los posibles sesgos del método empleado y se comparan los resultados con los obtenidos por la lectura de otolitos y otros AFT tanto en el Perú como en Chile.

\section{Material y métodos}

Estimación de las frecuencias por tallas.- Para la estimación de las frecuencias de tallas se utilizó la información proveniente de 24612 muestreos biométricos realizados por el Programa de Seguimiento de la Pesquería Pelágica del IMARPE en los principales puertos de desembarque del litoral peruano. Las muestras de T. murphyi provinieron de las capturas de la flota artesanal y la flota de cerco realizadas entre enero de 1972 y abril del 2012 (Fig. 1). Hasta mediados de la década de 1970 las muestras provinieron principalmente de las capturas de la flota artesanal y de la flota de cerco dedicada al consumo humano directo. Desde fines de la década de 1970 los muestreos de T. murphyi se concentraron principalmente en las capturas de la flota de cerco industrial dedicada a la pesca para la producción de harina y aceite de pescado. 
Cada muestreo biométrico correspondió a un viaje de pesca y las frecuencias resultantes fueron ponderadas a la captura del viaje respectivo antes de ser agrupadas por mes y por año. Para el agrupamiento anual se utilizó el ańo biológico que, de acuerdo al patrón reproductivo (Perea et al. 2013) abarca de noviembre de un año a octubre del año siguiente. Para las ponderaciones se utilizaron los parámetros de la relación talla-peso del año respectivo. Este proceso permitió la construcción de 446 vectores mensuales y 41 anuales. La Figura 2 muestra los histogramas de las frecuencias por tallas acumuladas anualmente a manera de resumen para todo el periodo de estudio. El rango de tallas observado abarcó de 5 a $79 \mathrm{~cm}$ de longitud total.

Estimación de parámetros mediante el ajuste de curvas de crecimiento a progresiones modales.- La estimación de los parámetros de crecimiento consistió en la identificación y trazado de curvas de crecimiento a través de una sucesión de frecuencias por tallas secuencialmente ordenadas en el tiempo. Para ello se utilizó el modelo generalizado de crecimiento de von Bertalanffy (Pauly \& Gaschuts 1979):

$$
\mathrm{L}_{\mathrm{t}}=\mathrm{L}_{\infty}\left(1-\exp -\left(\mathrm{k}\left(\mathrm{t}-\mathrm{t}_{0}\right)+\left(\mathrm{C}_{\mathrm{k}} / 2 \pi\right) \sin 2 \pi\left(\mathrm{t}-\mathrm{t}_{\mathrm{s}}\right)\right)\right)
$$

Donde $\mathrm{L}_{\mathrm{t}}$ es la talla a la edad, $\mathrm{L}_{\infty}$ es la longitud asintótica, $\mathrm{k}$ es la constante de crecimiento, $\mathrm{t}_{0}$ es la edad teórica a la cual el pez tendría una talla igual a cero centímetros, $\mathrm{C}_{\mathrm{k}}$ denota la amplitud de la oscilación, $\mathrm{t}_{\mathrm{s}}$ es el Summer Point o momento del año en que el crecimiento es más rápido. El $t_{s}$ está relacionado al "Winter Point" a través de WP $=\mathrm{t}_{\mathrm{s}}+0.5$.

Para la estimación de los parámetros se utilizó la rutina ELEFAN I (Pauly \& David 1981, Pauly 1987) del programa FISAT II (Gayanilo et al. 2005). De manera resumida, el procedimiento utilizado por el ELEFAN I consiste en: (1) reestructurar las frecuencias por tallas del arreglo temporal con el propósito de resaltar las marcas de clase con las frecuencias más altas; (2) construir de manera iterativa un conjunto de curvas de crecimiento, a partir de la proposición arbitraria de combinaciones de valores de $\mathrm{L}_{\infty}, \mathrm{k}, \mathrm{C}$ y WP; (3) hacer pasar las curvas por el arreglo de las frecuencias por tallas; (4) asignar a cada curva un puntaje cada vez que pase por una marca de clase resaltada; $y,(5)$ estimar el puntaje acumulado (score) obtenido por cada curva luego de haber pasado por todas la frecuencias del arreglo. Dadas las frecuencias por tallas analizadas, el programa sugiere como los parámetros más probables aquellos con los que se construye la curva capaz de pasar por la mayor cantidad de marcas resaltadas (i. e. curva con el score más alto).

Debido a los cambios en estructura de la flota y los cambios en la abundancia y disponibilidad de T. murphyi, en los últimos ańos se han dejado de observar las tallas más grandes que si estaban presentes en las capturas hasta inicios de la década de 1970. Por ello se utilizó la ecuación empírica propuesta por Froese \& Binohlan (2000) para calcular la longitud asintótica $\left(\mathrm{L}_{\infty}\right)$ a partir de la máxima talla máxima $\left(\mathrm{L}_{\max }\right)$ observada en la pesquería:

$$
\log _{10}\left(\mathrm{~L}_{\infty}\right)=0.044+0.9841 * \log _{10} \mathrm{~L}_{\max } \pm 0.0741
$$

El parámetro $\mathrm{L}_{\infty}$ así calculado se mantuvo constante para la estimación del parámetro k. De esta manera se pudo utilizar las progresiones modales de aquellos periodos en los cuales no se observaron tallas grandes en los muestreos de la flota industrial de cerco sin el riesgo de sobrestimar la tasa de crecimiento $\mathrm{k}$.

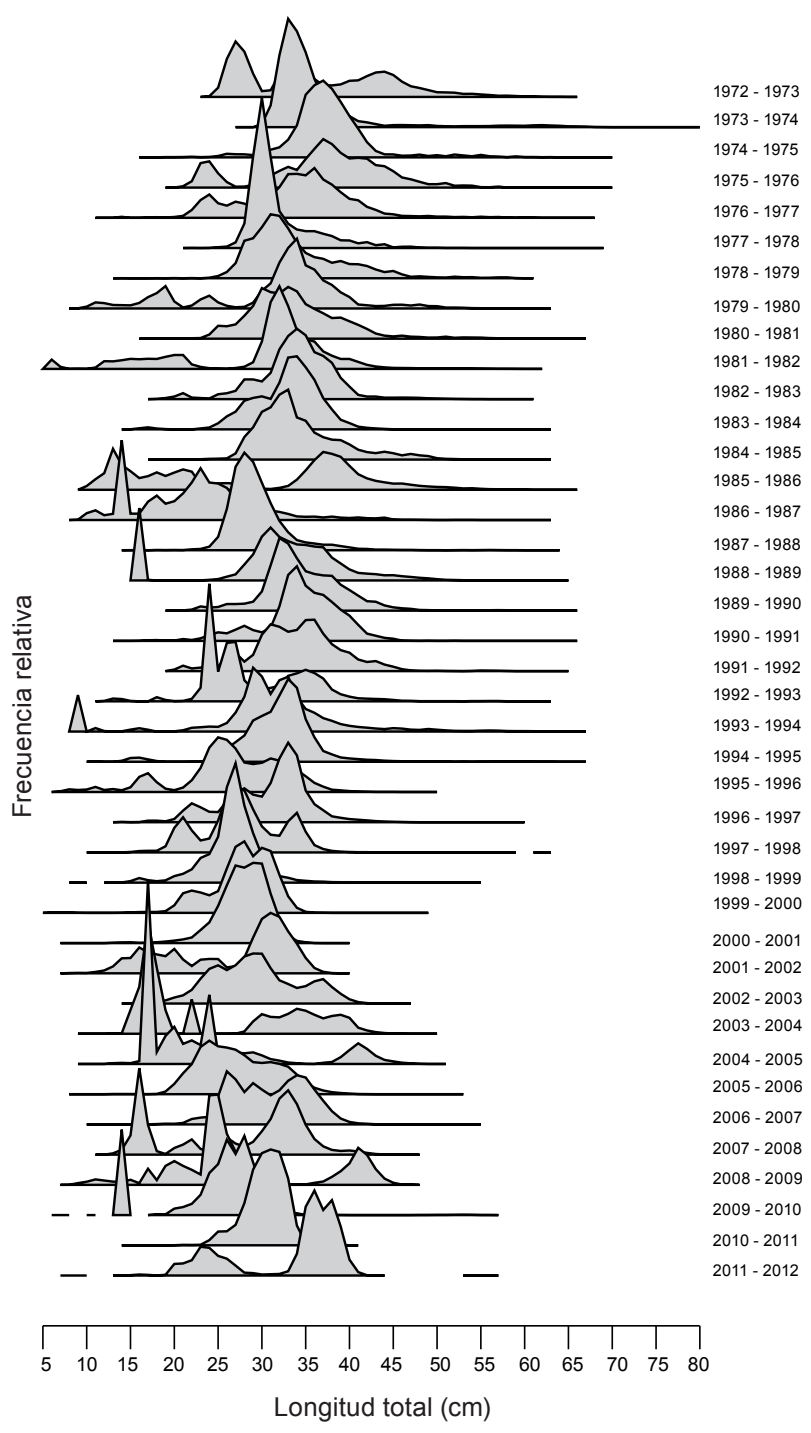

Figura 2. Frecuencias por tallas anuales del jurel T. murphyi capturado en el Perú entre los años biológicos 1972-73 y 2011-12

Figure 2. Annual length frequencies of Jack mackerel T. murphyi caught in Peru in biological years 1972-73 to 2011-12

Con el propósito de tener la mejor resolución posible para el seguimiento de los grupos modales se hizo el análisis en base a las frecuencias de tallas a escala mensual. En los primeros años (1972 - 1993) no se observó una clara progresión modal, por lo tanto, se hizo el análisis para el periodo comprendido entre 1994 y el 2012 donde si fue posible seguir grupos modales de manera clara e ininterrumpida.

\section{Resultados}

La $\mathrm{L}_{\text {max }}$ observada a lo largo de todo el periodo de estudio fue de $79 \mathrm{~cm}$ (febrero de 1974) y la $\mathrm{L}_{\infty}$ estimada a partir de la relación empírica de Froese $\&$ Binohlan (2000) fue de $81.6 \mathrm{~cm}$, con un límite inferior de $68.8 \mathrm{~cm}$ y uno superior de $96.7 \mathrm{~cm}$.

Para el periodo 1992 - 2012 los valores de $\mathrm{k}$ fluctuaron entre 0.15 y $0.18 \mathrm{año}^{-1}$, con una media de $0.167 \pm 0.012 \mathrm{año}^{-1}$. Los valores de $\mathrm{k}$ más bajos $\left(0.15 \mathrm{año}^{-1}\right)$ fueron estimados para las progresiones que predominaron durante los años 1993 y 2000. Los valores más altos $\left(0.18\right.$ ańo $\left.^{-1}\right)$ fueron estimados para las progresiones de los años 1998, 1999 y 2003 - 2004. Los valores de WP fluctuaron entre 0.17 y 0.75 , mientras que los de $\mathrm{C}$ entre 0.6 y 1.0. La progresión con la bondad de ajuste más baja (score 
Tabla 1. Estimados de k, WP, C y score de la bondad de ajuste para los grupos de edad seguidos durante el periodo $1993-2012$.

Table 1. Estimated k, WP, C and goodness of fit scores for the age groups followed during the period $1993-2012$.

\begin{tabular}{cccccc}
\hline $\begin{array}{c}\text { Grupo de edad } \\
\text { seguido durante }\end{array}$ & $\begin{array}{c}\text { Periodo de pre- } \\
\text { dominancia }\end{array}$ & $\begin{array}{c}\mathbf{k} \\
\text { (año }^{-1} \text { ) }\end{array}$ & WP & C & Score \\
\hline $1992-1994$ & 1993 & 0.15 & 0.50 & 0.9 & 0.150 \\
$1994-1995$ & 1995 & 0.17 & 0.75 & 0.8 & 0.135 \\
$1994-1997$ & 1996 & 0.16 & 0.75 & 0.7 & 0.126 \\
$1998-1999$ & 1998 & 0.18 & 0.25 & 0.6 & 0.152 \\
$1998-2000$ & 1999 & 0.18 & 0.58 & 0.9 & 0.136 \\
$1999-2001$ & 2000 & 0.15 & 0.58 & 0.6 & 0.128 \\
$2000-2003$ & 2001 & 0.16 & 0.17 & 0.9 & 0.130 \\
$2002-2005$ & $2003-2004$ & 0.18 & 0.08 & 0.9 & 0.131 \\
$2005-2008$ & $2006-2007$ & 0.17 & 0.58 & 1.0 & 0.193 \\
$2010-2012$ & $2011-2012$ & 0.17 & 0.58 & 0.6 & 0.220 \\
\hline & Promedio & 0.167 & & &
\end{tabular}

$=0.126)$ fue la que predominó durante 1996, mientras que la que presentó la bondad de ajuste más alta $($ score $=0.220)$ fue la predominante durante el periodo $2011-2012$ (Fig. 3, Tabla 1).

\section{Discusión}

En este trabajo se prefirió usar la ecuación de Froese \& Binohlan (2000) para calcular $\mathrm{L}_{\infty}$ dado que esta relación empírica basada en el análisis de más de 550 observaciones sobre diferentes especies, diferentes stocks de una misma especie y diferentes sexos de un mismo stock permite calcular límites de confianza, aunque la conocida aproximación $\mathrm{L}_{\text {max }} / \mathrm{L}_{\infty}$ (Pauly 1983, después de Taylor 1958) da valores semejantes.

Para la estimación del parámetro de crecimiento k se estimó primero $\mathrm{L}_{\infty}$ y se le mantuvo fijo para todo el periodo de estudio, esto con la finalidad de utilizar las progresiones modales de los últimos ańos, en los cuales no se observaron tallas grandes en los muestreos de la flota industrial de cerco, sin el riesgo de sobrestimar la tasa de crecimiento $\mathrm{k}$. Al respecto, cabe destacar que independientemente del método empleado, las estimaciones de $\mathrm{L}_{\infty}$ a partir de frecuencias de talla sin presencia de tallas mayores suele resultar en la subestimación de éste parámetro y la sobreestimación de $\mathrm{k}$ dada la relación inversa entre estos parámetros (Quinn \& Deriso 1999). Además, existe alguna evidencia que sugiere que el parámetro $\mathrm{L}_{\infty}$ sería más estable que el parámetro k (Isaac 1990).

La ausencia de individuos de mayor tamaño en las muestras recientes puede ser explicada por diferentes factores (Chen $\&$ Watanabe 1989, Lorenzen 1996, Pauly \& Morgan eds. 1987), entre los cuales cabe destacar los cambios ambientales de corto, mediano y largo plazo, los cambios en la abundancia, distribución y disponibilidad, y los cambios en las regulaciones pesqueras y en la estructura y operación de la flota (Flores et al. 2013, Espino 2013, Dioses 2013b, Segura \& Aliaga 2013, Zuzunaga 2013, Niquen et al. 2013).

En cuanto al ajuste de curvas de crecimiento a las frecuencias por tallas, se consideró apropiado iniciar el proceso a partir del periodo de tiempo en que se evidenciaron las progresiones modales (1993). Una de las reconocidas limitaciones de los AFT y en especial del ELEFAN I es la imposibilidad de estimar el crecimiento a partir de frecuencias por tallas que no evidencian una sucesión temporal de modas (Pauly \& David 1981). Dichas progresiones no fueron evidentes en el periodo 1972 - 1992, por lo que forzar el ajuste de curvas de crecimiento a estas frecuencias con modas casi constantes hubiera dado resultados contradictorios, poco consistentes y con estimaciones de $\mathrm{k}$ poco realistas.

Es importante destacar la similitud existente entre los parámetros de crecimiento estimados para el T. murphyi capturado en el Perú (métodos del M2 al M4, Tabla 2), sobre todo si tenemos en cuenta que las estimaciones han sido realizadas utilizando metodologías independientes tanto en lo referente a las fuentes de información como a los procedimientos. Los valores de k son muy próximos entre sí, pero consistentemente más altos que los estimados para el T. murphyi capturado frente

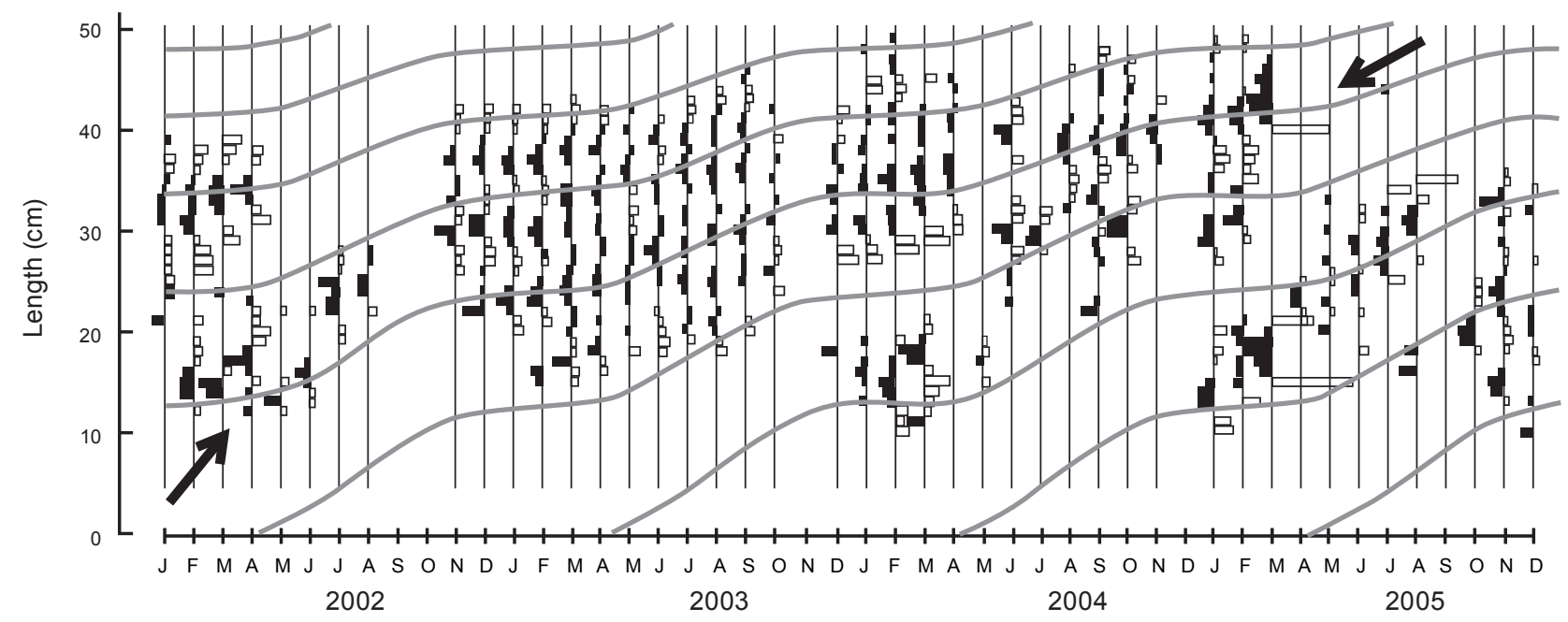

Figura 3. Ajuste de una curva de crecimiento a la progresión de modas que pudo ser seguida desde enero del 2002 (flecha izquierda-derecha, moda en $12 \mathrm{~cm}$ ) hasta marzo del 2005 (flecha derecha-izquierda, moda en $40 \mathrm{~cm}$ ).

Figure 3. Fitting of a growth curve to the modal progression followed from January 2012 (left-right arrow, mode in $12 \mathrm{~cm}$ ) to March 2005 (rightleft mode, mode in $40 \mathrm{~cm}$ ). 
Tabla 2. Comparación de los parámetros de crecimiento estimados por diferentes autores para el jurel T. murphyi capturado frente al Perú y Chile. Table 2. Comparison of the growth parameters estimated by different authors and methods for the Jack mackerel T. murphyi caught off Peru and Chile.

\begin{tabular}{|c|c|c|c|c|c|c|}
\hline Método & Autor & Lugar & Base del Método & $L_{\infty}(\mathrm{cm})$ & $K$ & $t_{0}$ \\
\hline M1 & Díaz (2013) & Perú & $\begin{array}{l}\text { Relación empírica (Froese } \\
\quad \text { \& Binohlan. 2000) }\end{array}$ & 81.6 & & \\
\hline M2 & Díaz (2013) & Perú & AFT (ELEFAN I) & & 0.167 & \\
\hline M3 & Goicochea et al. (2013) & Perú & Lectura de otolitos & 75.17 & 0.165 & -0.817 \\
\hline M4 & Dioses (1995) & Perú & Lectura de otolitos & 80.8 & 0.155 & -0.356 \\
\hline M5 & Cubillos \& Arancibia (1995) & Chile (Centro-Sur) & AFT (ELEFAN) & $78.6^{*}$ & 0.138 & \\
\hline M6 & Gili et al. (1995) en Serra \& Canales 2007 & Chile (Centro-Sur) & Lectura de otolitos & $77.8^{*}$ & 0.094 & -0.896 \\
\hline
\end{tabular}

* Convertidas de $\mathrm{L}_{\infty}$ a la horquilla $\left(\mathrm{Lh}_{\infty}\right)$ a $\mathrm{L}_{\infty}$ total $\left(\mathrm{Lt}_{\infty}\right)$ a partir de la relación $\mathrm{L}_{\mathrm{t}}=0,514+1,091 \mathrm{LH}$ (Cubillos \& Arancibia 1995)

a Chile (métodos M5 y M6, Tabla 2). Esto sugiere que frente al Perú el T. murphyi tendría una tasa de crecimiento más alta que frente a Chile.

Existen múltiples ejemplos de especies donde sus diversos stocks tienen parámetros de crecimiento diferentes y que se explicarían por estar expuestas a distintas condiciones ambientales (Jennings \& Beverton 1991, Ormseth \& Norcross 2009), niveles de explotación (Law 2000) o estructuras comunitarias diferentes (Gislason et al. 2008).

Por las facilidades para su aplicación, consideramos que el método empleado en este estudio podría ser utilizado de forma rutinaria para futuras estimaciones del crecimiento del T. murphyi en todo el ámbito de distribución de la especie en el Pacífico Sur. Sin embargo, también es recomendable utilizar otros métodos y sugerimos que en futuros estudios de la edad y crecimiento del T. murphyi en el Pacífico Sudeste se incluyan: 1) estimaciones a partir de uno o más de los diferentes metodos de AFT disponibles, tanto paramétricos (e.g. MULTIFAN, NORMSEP) como no paramétricos (e.g. SLCA, PROJMAT, Powell-Wetherall); 2) análisis de sensibilidad de los resultados a los datos de entrada; 3) análisis de las potencialidades y debilidades de cada método, medidos a través de indicadores de desempeño; y, 4) una comparación final de todos los resultados. Otro aspecto que no se debe dejar de lado es procurar que el muestreo siempre sea representativo tanto en términos de espacio como de tiempo.

\section{Agradecimientos}

El autor desea expresar su más sincero agradecimiento a la Dirección General de Investigaciones en Recursos Pelágicos del IMARPE por las facilidades otorgadas para la preparación de este trabajo, a Ricardo Oliveros-Ramos y al revisor anónimo por los aportes al trabajo y a Giancarlo Morón por el apoyo computacional y de revisión bibliográfica.

\section{Literatura citada}

Abramov A. \& A. Kotlyar. 1980. Some biological features of the Peruvian Jack mackerel, Trachurus murphyi Nichols, 1920. Invest. Mar. 6(6):89-100.
Cubillos L. \& H. Arancibia. 1995. Comparative growth performance of horse mackerel of the genus Trachurus, with emphasis on T. symmetricus murphyi in Chile. Sci. Mar. 59(3-4):647-652.

Chen S. \& S. Watanabe. 1989. Age dependence of natural mortality coefficient in fish population dynamics. Bulletin of the Japanese Society of Scientific Fisheries 55(2): 205-208.

Dioses T. 1995. Análisis de la distribución y abundancia de los recursos jurel y caballa frente a la costa peruana. Informe Progresivo del Instituto del Mar del Perú (IMARPE), Callao, Perú, 1995, N³, 55 p.

Dioses T. 2013a. Edad y crecimiento del jurel Trachurus murphyi en el Perú. En: Csirke J., R. Guevara-Carrasco \& M. Espino (Eds.). Ecología, pesquería y conservación del jurel (Trachurus murphyi) en el Perú. Rev. peru. biol. número especial 20(1): 045 - 052

Dioses T. 2013b. Patrones de distribución y abundancia del jurel Trachurus murphyien el Perú. En: Csirke J., R. Guevara-Carrasco \& M. Espino (Eds.). Ecología, pesquería y conservación del jurel (Trachurus murphyi) en el Perú. Rev. peru. biol. número especial 20(1): $067-074$

Espino M. 2013. El jurel Trachurus murphyi y las variables ambientales de macroescala. En: Csirke J., R. Guevara -Carrasco \& M. Espino (Eds.). Ecología, pesquería y conservación del jurel (Trachurus murphyi) en el Perú. Rev. peru. biol. número especial 20(1): $009-020$

Flores R., M. Espino, G. Luque \& J. Quispe. 2013. Patrones de variabilidad ambiental en el mar peruano. En: Csirke J., R. Guevara-Carrasco \& M. Espino (Eds.). Ecología, pesquería y conservación del jurel (Trachurus murphyi) en el Perú. Rev. peru. biol. número especial 20(1): $021-028$

Froese R. \& C. Binohlan. 2000. Empirical relationships to estimate asymptotic length, length at first maturity and length at maximum yield per recruit in fishes, with a simple method to evaluate length frequency data. Journal of Fish Biology 56(4): 758-773. DOI: 10.1111/j.1095-8649.2000.tb00870.x

Gayanilo Jr. F., P. Sparre \& D. Pauly. 2005. The FAO-ICLARM Stock Assessment Tools II Windows Version (FISAT II) user's guide (Revision I). FAO Computerized Information Series Fisheries, $N^{\circ} 8$.

Gislason H., J. Pope, J. Rice, \& N. Daan. 2008. Coexistence in North Sea fish communities: implications for growth and natural mortality. - ICES Journal of Marine Science, 65: 514-530. DOI: 10.1093/ icesjms/fsn035

Goicochea C., J. Mostacero P. Moquillaza, T. Dioses, Y. Topiño \& Renato Guevara-Carrasco. 2013. Validación de los anillos de crecimiento de otolitos del jurel Trachurus murphyi. En: Csirke J., R. GuevaraCarrasco \& M. Espino (Eds.). Ecología, pesquería y conservación del jurel (Trachurus murphyi) en el Perú. Rev. peru. biol. número especial 20(1): $053-060$ 
Gulland J. 1987. Length-based methods in fisheries research: from theory to application, p. 335-342. In D. Pauly and G.R. Morgan (eds.) Length-based methods in fisheries research. ICLARM Conference Proceedings 13, 468 p. International Center for Living Aquatic Resources Management, manila, Philippines, and Kuwait Institute for Scientific Research, Safat, Kuwait.

Isaac V. 1990. The accuracy of some length-based methods for fish population studies. ICLARM Tech. Rep. 27.81 p.

Jennings S. \& R. Beverton. 1991. Intraspecific variation in the life history tactics of Atlantic herring (Clupea harengus L.) stocks. ICES Journal of Marine Science, 48: 117 -125. DOI: 10.1093/icesjms/48.1.117

Kochkin P. 1994. Age determination and estimate of growth rate for the Peruvian Jack mackerel Trachurus symmetricus murphyi. J. Ichthyol. 34(3):39-50.

Law R. 2000. Fishing, selection, and phenotypic evolution. - ICES Journal of Marine Science, 57: 659-669. DOI: 10.1006/jmsc.2000.0731

Lorenzen K. 1996. The relationship between body weight and natural mortality in juvenile and adult fish: a comparison of natural ecosystems and aquaculture. Journal of Fish Biology 49:627-647. DOI: 10.1111/j.1095-8649.1996.tb00060.x

Ñiquen M., M. Bouchon, D. Ulloa \& A. Medina. 2013. Análisis de la pesquería del jurel Trachurus murphyi en el Perú. En: Csirke J., R. GuevaraCarrasco, M. Espino (Eds.). Ecología, pesquería y conservación del jurel (Trachurus murphyi) en el Perú. Rev. peru. biol. número especial 20(1): 097- 106

Ormseth O. \& B. Norcross. 2009. Causes and consequences of life-history variation in North American stocks of Pacific cod. - ICES Journal of Marine Science, 66: 349-357. DOI: 10.1093/icesjms/fsn 156

Pauly D. 1983. Some simple methods for the 1983 assessment of tropical fish stocks. FAO Fish.Tech.Pap., 234:52 p.

Pauly D. 1987. A review of the ELEFAN system for analysis of length-frequency data in fish and aquatic invertebrates, p. 7-34. In D. Pauly and G.R. Morgan (eds.) Length-based methods in fisheries research. ICLARM Conference Proceedings 13, 468 p. International Center for Living Aquatic Resources Management, manila, Philippines, and Kuwait Institute for Scientific Research, Safat, Kuwait.
Pauly D. \& N. David. 1981. ELEFAN I, a BASIC program for the objective extraction of growth parameters from length-frequency data. Berichte der Deutschen Wissensschaftlichen Kommission für Meeresforschung 28(4): 205-211.

Pauly, D. \& G. Gaschuts. 1979. A simple method for fitting oscillating growth data, with a program for pocket calculators. ICES C. M. 1979/G: 24. 26 pp.

Pauly D. \& G. Morgan (Eds.) 1987. Length-based methods in fisheries research. ICLARM Conference Proceedings 13, 468 p. International Center for Living Aquatic Resources Management, manila, Philippines, and Kuwait Institute for Scientific Research, Safat, Kuwait.

Perea A., J. Mori, B. Buitrón \& J. Sánchez. 2013. Aspectos reproductivos del jurel Trachurus murphyien el Perú. En: Csirke J., R. Guevara -Carrasco \& M. Espino (Eds.). Ecología, pesquería y conservación del jurel (Trachurus murphyi) en el Perú. Rev. peru. biol. número especial 20(1): $029-034$

Quinn T. \& R. Deriso. 1999. Quantitative fish dynamics. Oxford University Press, New York, N.Y. pp: 560.

R. 2012. The R Foundation for Statistical Computing. R version 2.15.1

Segura M. \& A. Aliaga M. 2013. Biomasa acústica y distribución del jurel Trachurus murphyien el Perú. En: Csirke J., R. Guevara -Carrasco \& M. Espino (Eds.). Ecología, pesquería y conservación del jurel (Trachurus murphyi) en el Perú. Rev. peru. biol. número especial 20(1): $087-096$

Serra R. \& C. Canales. 2009. Short review of some biological aspects of the Chilean Jack mackerel, Trachurus murphyi. SP-07-SWG-JM-SA-05, 7p.

Taylor C.C. 1958. Cod growth and temperature. J. Cons. perm. Int. Explor. Mer., 23(3): 365-370

Zuzunaga J. 2013. Medidas de conservación y ordenación pesquera del jurel Trachurus murphyi en el Perú. En: Csirke J., R. Guevara -Carrasco, M. Espino (Eds.). Ecología, pesquería y conservación del jurel (Trachurus murphyi) en el Perú. Rev. peru. biol. número especial 20(1): 097- 106 